\section{(2) OPEN ACCESS}

\title{
Changes in prolonged sedentary behaviour across the transition to retirement
}

\author{
Kristin Suorsa (1) , ${ }^{1,2}$ Anna Pulakka (1) , ${ }^{3}$ Tuija Leskinen, ${ }^{1,2}$ Jaana Pentti, ${ }^{1,4}$ \\ Jussi Vahtera (1) , ${ }^{1,2}$ Sari Stenholm (1) 1,2
}

- Additional material is published online only. To view, please visit the journal online (http://dx.doi.org/10.1136/ oemed-2020-106532).

${ }^{1}$ Department of Public Health, University of Turku, and Turku University Hospital, Turku, Finland

${ }^{2}$ Centre for Population Health Research, University of Turku, and Turku University Hospital, Turku, Finland

${ }^{3}$ Department of Public Health Solutions, Public Health Promotion Unit, Finnish Institute for Health and Welfare, Helsinki, Uusimaa, Finland

${ }^{4}$ Clinicum, Faculty of Medicine, University of Helsinki, Helsinki, Finland

\section{Correspondence to}

Kristin Suorsa, Department of Public Health, University of Turku, 20520 Turku, Finland; kristin.suorsa@utu.fi

Received 9 March 2020 Revised 10 September 2020 Accepted 24 September 2020 Published Online First 17 November 2020

\section{ABSTRACT}

Background Prolonged sedentary behaviour is associated with a higher risk of cardiometabolic diseases. This longitudinal study examined changes in daily total, prolonged ( $\geq 30 \mathrm{~min}$ ) and highly prolonged ( $\geq 60 \mathrm{~min}$ ) sedentary time across the transition to retirement by gender and occupational status.

Methods We included 689 aging workers (mean (SD) age before retirement 63.2 (1.6) years, 85\% women) from the Finnish Retirement and Aging Study (FIREA). Sedentary time was measured annually using a wrist-worn triaxial ActiGraph accelerometer before and after retirement with on average 3.4 (range 2-4) measurement points.

Results Women increased daily total sedentary time by $22 \mathrm{~min}(95 \% \mathrm{Cl} 13$ to 31), prolonged sedentary time by $34 \mathrm{~min}(95 \% \mathrm{Cl} 27$ to 42$)$ and highly prolonged sedentary time by $15 \mathrm{~min}(95 \% \mathrm{Cl} 11$ to 20$)$ in the transition to retirement, and remained at the higher level of sedentary time years after retirement. The highest increase in total and prolonged sedentary time was observed among women retiring from manual occupations. Men had more total and prolonged sedentary time compared with women before and after retirement. Although no changes in men's sedentary time were observed during the retirement transition, there was a gradual increase of $33 \mathrm{~min}(95 \% \mathrm{Cl} 6$ to 60$)$ in prolonged sedentary time from pre-retirement years to post-retirement years.

Conclusion The transition to retirement was accompanied by an abrupt increase in prolonged sedentary time in women but a more gradual increase in men. The retirement transition may be a suitable time period for interventions aiming to decrease sedentary behaviour.

\section{INTRODUCTION}

High levels of sedentary behaviour are associated with chronic diseases and mortality. ${ }^{1}$ Moreover, accumulation of sedentary time in uninterrupted, prolonged bouts is dose-dependently associated with higher cardiovascular disease risk, ${ }^{2}$ and especially sedentary bouts lasting $\geq 30 \mathrm{~min}$ in comparison to shorter bouts have been linked to greater all-cause mortality. ${ }^{3}$

We have shown previously that accelerometermeasured daily total sedentary time increases in the transition to retirement, ${ }^{4}$ but it is not known whether increased sedentary time includes changes in harmful prolonged sedentary time and how long the changes persist. It has been shown that

\section{Key messages}

What is already known about this subject?

- Accelerometer-measured prolonged sedentary time is higher on workdays compared to days off, especially among office workers.

- Sedentary time seems to increase after the transition to retirement, especially among women retiring from manual occupations.

- No previous studies have examined how prolonged sedentary time changes in the transition to retirement and how long the observed changes persist.

What are the new findings?

- Retiring women increased total and prolonged sedentary time after the transition to retirement and the level was maintained about 2 years after the retirement.

- Men had notably more sedentary time compared to women before and after the transition to retirement.

- Women retiring from manual occupations increased their prolonged sedentary time more than women retiring from non-manual occupations.

How might this impact on policy or clinical practice in the foreseeable future?

- Since prolonged sedentary time is associated with harmful health consequences, retirees should be encouraged to break up prolonged sitting.

- The transition to retirement could be a suitable time period for interventions to decrease sedentary time.

prolonged sedentary behaviour is more prominent on workdays compared with days off, especially among office workers. ${ }^{5} 6$ Thus, it is possible that prolonged sedentary time decreases after retirement when there is more time for activities not related to work or passive commuting. On the other hand, increased time spent at home may include passive activities such as watching television, which may in turn increase prolonged sedentary time. ${ }^{7}$

The aim of this study was to examine changes in daily total and prolonged sedentary time across the retirement transition by following aging workers with annual accelerometer measurements 
from final years at work to a few years after the statutory retirement.

\section{METHODS}

\section{Study population}

This study is based on the Finnish Retirement and Aging Study (FIREA) which is an ongoing longitudinal cohort study of retiring municipal workers in Finland established in 2013, ${ }^{8}$ described previously in detail. ${ }^{4}$

Between September 2014 and March 2020, 689 of the 908 eligible participants who had given written informed consent had successfully worn the accelerometer immediately before and after the transition to full-time statutory retirement. The rest of the participants were not yet retired $(n=197)$, did not wear the accelerometer $(n=13)$, or wore the accelerometer but had $<4$ valid measurement days either before or after transition to retirement $(n=9)$, and were therefore excluded from the analyses. The average number of measurement points was 3.4 (range 2-4; 1.7 before and 1.7 after retirement). The mean (SD) number of valid days was 6.8 days $(0.5)$ per participant at each wave.

\section{Accelerometer measurements}

Sedentary time was measured with wrist-worn triaxial ActiGraph wActiSleep-BT and wGT3X-BT accelerometers (ActiGraph, Pensacola, Florida, USA). Detailed measurement and data reduction procedures are described in our previous work. ${ }^{4}$ Briefly, participants wore accelerometers on their non-dominant wrist for 7 consecutive days and nights once a year, with a mean of 361-364 days between the consecutive waves. Sleep time was excluded by the algorithm available in the ActiLife software ${ }^{9}$ and non-wear time by the Choi algorithm. ${ }^{10}$ Only valid days including $\geq 10$ hours of wake wear time were included in the analyses. ${ }^{4}$ We defined sedentary time using a cutpoint of $<1853$ vector magnitude counts per min, validated against a thigh-worn accelerometer among older adults in free-living conditions, ${ }^{11}$ and defined sedentary bout as consequent minutes spent sedentary ending to $a \geq 1$ min break spent in non-sedentary activity. ${ }^{2}$ We calculated daily means of total sedentary time and time spent in prolonged ( $\geq 30 \mathrm{~min}$ ) and in highly prolonged $(\geq 60 \mathrm{~min}$ ) sedentary bouts at each study wave before (waves -2 and -1 ) and after the transition to retirement (waves +1 and +2 ).

\section{Assessment of covariates}

Gender, date of birth and occupational status were obtained from the Keva register. ${ }^{8}$ Occupational status was categorised based on the International Standard Classifications of Occupations (ISCO) ${ }^{12}$ into non-manual (ISCO classes 1-4) and manual workers (ISCO classes 5-9) according to the last known occupation preceding retirement.

Smoking status (never/former and current), body mass index (under/normal weight, overweight and obese), number of chronic diseases $(0,1$ and $\geq 2)$ and mobility limitations (limitations in walking $2 \mathrm{~km}$ : none, minor and major ${ }^{13}{ }^{14}$ ) were derived from the questionnaires immediately before retirement (wave -1 ). ${ }^{4}$

\section{Statistical analyses}

The characteristics of the study population before retirement are shown as percentages for categorical variables and means and SD for continuous variables.

To illustrate daily total sedentary time, prolonged and highly prolonged sedentary time by gender before and after the transition to retirement, we used linear mixed models by adjusting for wake wear time. We also compared daily sedentary time, prolonged and highly prolonged sedentary time in the transition to retirement, that is, immediately before (wave -1) and after retirement (wave +1 ), by gender and occupational status using linear mixed models and adjusting for confounding factors.

All statistical analyses were performed using SAS statistical software, version 9.4 (SAS Institute, Inc, Cary, NC, USA).

\section{RESULTS}

The characteristics of the study population immediately before retirement are presented in online supplemental table 1 . The mean (SD) age was 63.2 (1.6) years for the women and 63.3 (1.4) years for the men. The majority of the participants were women (85\%) and non-manual workers (66\%).

In women, daily total sedentary time, as well as prolonged and highly prolonged sedentary time, did not change notably before retirement but increased markedly in the transition to retirement $(p<0.0001)$ and levelled off after retirement (figure 1). In the transition to retirement, the observed increase was 22 minutes (95\% CI 13 to 31 ) in daily total sedentary time, 34 minutes $(95 \%$ CI 27 to 42) in prolonged sedentary time and $15 \mathrm{~min}$ (95\% CI 11 to 20 ) in highly prolonged sedentary time (online supplemental table 2). In particular, women retiring from manual occupations increased their total and prolonged sedentary time (online supplemental table 2).

Men increased daily total sedentary time, as well as prolonged and highly prolonged sedentary time, in the year preceding retirement (21 $\mathrm{min}, 95 \%$ CI 6 to $35 ; 23 \mathrm{~min}, 95 \%$ CI 10 to 36 ;
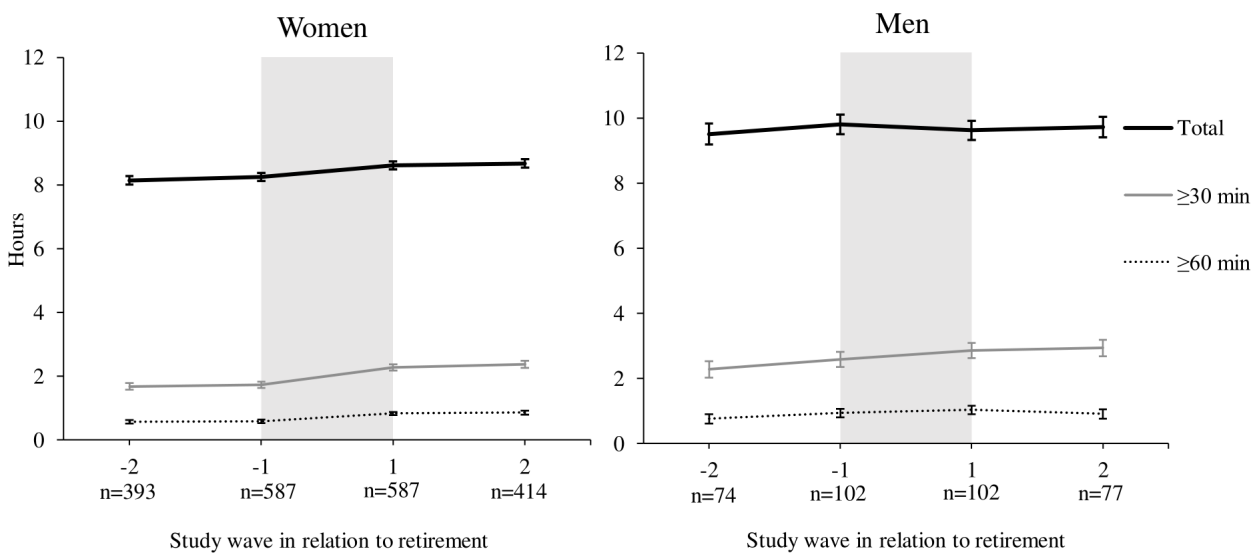

Figure 1 Total daily sedentary time, prolonged ( $\geq 30 \mathrm{~min}$ ) and highly prolonged ( $\geq 60 \mathrm{~min}$ ) sedentary time across the retirement transition in women and men. 
11 min, 95\% CI 2 to 19 , figure 1), but no statistically significant changes were observed during the transition to retirement (online supplemental table 2). An overall increase in prolonged sedentary time was observed from wave -2 to wave +2 (33 min, $95 \%$ CI 6 to 60 , figure 1). Men had significantly more daily total and prolonged sedentary time compared with women at all time points.

\section{DISCUSSION}

This longitudinal accelerometer-based study showed that the transition to retirement induced a notable increase in prolonged sedentary time in women. In men, prolonged sedentary time increased more gradually across the retirement transition.

To the best of our knowledge, this is the first longitudinal study to report changes in accelerometer-measured sedentary time from pre-retirement years to post-retirement years. Previous knowledge on long-term changes in sedentary time across the transition to retirement is based on self-reports, ${ }^{8}$ which cannot be used to examine sedentary bouts and are subject to recall and information bias. Previous accelerometer-based findings about comparison of daily sedentary time before and after retirement ${ }^{4}$ do not provide information on how long-lasting the observed increase in daily sedentary time is and whether the increase is induced by the transition to retirement itself or by other factors, such as aging. ${ }^{15}$ With annual accelerometer measurements, we were able to show that the transition to retirement induced changes in sedentary behaviour in women, and especially changes in prolonged sedentary time were observed.

Our results extend previous knowledge by showing that previously observed higher daily total sedentary time after retirement $^{4}$ concerns particularly prolonged sedentary time, which is more harmful for health compared with short sedentary bouts. ${ }^{3}$ Interestingly, prolonged sedentary time did not decrease, but actually increased in the transition to retirement among those retiring from non-manual occupations, even though previous findings have shown that workdays include more prolonged sedentary time compared with days off, especially among office workers. ${ }^{5}{ }^{6}$ As a possible explanation, retirement generally brings changes to daily routines and social interactions, and the amount of active social participation after retirement may partly explain the amount of sedentary time. ${ }^{16}$ Social connections and meaningful activities may decrease after retirement, leading to increased time spent at home and engagement in sedentary activities such as watching television, which is likely done in a more prolonged manner than other sedentary activities such as using a computer. ${ }^{7}$ Moreover, when people retire, physical activity during commuting and lunch breaks no longer interrupt the periods of sitting .

Since an increase in prolonged sedentary behaviour increases the risk of cardiovascular disease and mortality dosedependently, ${ }^{23}$ retirees should be encouraged to break up sedentary activities. As men accumulated high sedentary time, they could especially benefit from interventions aiming to decrease sedentary time already in work-life. Future research on the health consequences related to increased sedentary time after retirement is needed.

The strengths of our study include a longitudinal study design, accelerometer-measured sedentary time and consideration of several individual characteristics associated with sedentary behaviour. ${ }^{16}$ The measurements were conducted at the same time of the year for each individual and therefore bias associated with seasonal variation was minimised. As a limitation, wrist-worn accelerometers may underestimate sedentary time, especially when compared with thigh-worn accelerometers. ${ }^{11}$ We used categorisation to non-manual and manual occupations as an indicator of work-related activity and socioeconomic status, but there may be heterogeneity in terms of sedentary behaviour within the occupational groups. Our study population comprised $85 \%$ women, which corresponds to the female-dominated target population of Finnish public sector workers. ${ }^{17}$

As there were no notable differences to the eligible study population, ${ }^{4}$ our results can be generalised to public sector employees in Finland or to countries with a similar statutory retirement age and pension system.

Acknowledgements The authors would like to thank all the participants who took part in the FIREA study and the FIREA study staff members for their contribution to the data collection.

Contributors SS and JV conceived and designed this study and the data collection. $\mathrm{KS}$, JP and AP analysed the data and KS drafted the manuscript, with critical revisions from SS, AP, TL, JP and JV. All authors approved the final version of the manuscript.

Funding This work was supported by the Academy of Finland (Grants 286294, 294154 and 319246 to SS; 309526 to TL); Finnish Ministry of Education and Culture (to SS), Nordforsk (to JV), Juho Vainio Foundation (to SS and KS), Päivikki and Sakari Sohlberg Foundation (to AP) and Hospital District of South-West Finland (to SS and AP).

Competing interests None declared.

Patient consent for publication Not required.

Ethics approval Ethics Committee of Hospital District of South-West Finland (84/1801/2014).

Provenance and peer review Not commissioned; externally peer reviewed.

Data availability statement Data are available upon reasonable request. Anonymised partial datasets of the Finnish Retirement and Aging Study are available by application from bona fide researchers with an established scientific record and bona fide organisations. For more information, please contact Professor Sari Stenholm (sari.stenholm@utu.fi).

Supplemental material This content has been supplied by the author(s). It has not been vetted by BMJ Publishing Group Limited (BMJ) and may not have been peer-reviewed. Any opinions or recommendations discussed are solely those of the author(s) and are not endorsed by BMJ. BMJ disclaims all liability and responsibility arising from any reliance placed on the content. Where the content includes any translated material, BMJ does not warrant the accuracy and reliability of the translations (including but not limited to local regulations, clinical guidelines, terminology, drug names and drug dosages), and is not responsible for any error and/or omissions arising from translation and adaptation or otherwise.

Open access This is an open access article distributed in accordance with the Creative Commons Attribution Non Commercial (CC BY-NC 4.0) license, which permits others to distribute, remix, adapt, build upon this work non-commercially, and license their derivative works on different terms, provided the original work is properly cited, appropriate credit is given, any changes made indicated, and the use is non-commercial. See: http://creativecommons.org/licenses/by-nc/4.0/.

\section{ORCID iDs}

Kristin Suorsa http://orcid.org/0000-0002-3520-8069

Anna Pulakka http://orcid.org/0000-0002-0602-8632

Jussi Vahtera http://orcid.org/0000-0002-6036-061X

Sari Stenholm http://orcid.org/0000-0001-7560-0930

\section{REFERENCES}

1 Patterson R, McNamara E, Tainio M, et al. Sedentary behaviour and risk of all-cause, cardiovascular and cancer mortality, and incident type 2 diabetes: a systematic review and dose response meta-analysis. Eur J Epidemio/ 2018;33:811-29.

2 Bellettiere J, LaMonte MJ, Evenson KR, et al. Sedentary behavior and cardiovascular disease in older women: the Objective Physical Activity and Cardiovascular Health (OPACH) study. Circulation 2019;139:1036-46.

3 Diaz KM, Howard VJ, Hutto B, et al. Patterns of sedentary behavior and mortality in U.S. middle-aged and older adults: a national cohort study. Ann Intern Med 2017:167:465-75.

4 Suorsa K, Pulakka A, Leskinen T, et al. Objectively measured sedentary time before and after transition to retirement: the Finnish Retirement and Aging Study. J Gerontol A Biol Sci Med Sci 2020;75:1737-43. 
5 Thorp AA, Healy GN, Winkler E, et al. Prolonged sedentary time and physical activity in workplace and non-work contexts: a cross-sectional study of office, customer service and call centre employees. Int J Behav Nutr Phys Act 2012;9:128.

6 Parry S, Straker L. The contribution of office work to sedentary behaviour associated risk. BMC Public Health 2013;13:296

7 Benatti FB, Ried-Larsen M. The effects of breaking up prolonged sitting time: a review of experimental studies. Med Sci Sports Exerc 2015;47:2053-61.

8 Leskinen T, Pulakka A, Heinonen OJ, et al. Changes in non-occupational sedentary behaviours across the retirement transition: the Finnish Retirement and Aging (FIREA) study. J Epidemiol Community Health 2018;72:695-701.

9 ActiGraph. What does the "Detect Sleep Periods" button do and how does it work? 2018, 2019. Available: https://actigraphcorp.force.com/support/s/article/What-doesthe-Detect-Sleep-Periods-button-do-and-how-does-it-work

10 Choi L, Ward SC, Schnelle JF, et al. Assessment of wear/nonwear time classification algorithms for triaxial accelerometer. Med Sci Sports Exerc 2012;44:2009-16.

11 Koster A, Shiroma EJ, Caserotti P, et al. Comparison of sedentary estimates between activPAL and hip- and wrist-worn ActiGraph. Med Sci Sports Exerc 2016;48:1514-22.
12 Statistics Finland. Classification of occupations. Available: http://www.stat.fi/meta/ luokitukset/ammatti/001-2010/index_en.html

13 Hays RD, Sherbourne CD, Mazel RM. The Rand 36-Item health survey 1.0. Health Econ 1993:2:217-27.

14 Aalto AM, Aro AR, Mähönen M. RAND 36-item health survey 1.0. Finnish version on the health-related quality of life questionnaire. Helsinki, Finland: Stakes, 1995.

15 Dogra S, Ashe MC, Biddle SJH, et al. Sedentary time in older men and women: an international consensus statement and research priorities. Br J Sports Med 2017:51:1526-32.

16 Chastin SFM, Buck C, Freiberger E, et al. Systematic literature review of determinants of sedentary behaviour in older adults: a DEDIPAC study. Int J Behav Nutr Phys Act 2015; 12:127.

17 Statistics Finland. The women and men in Finland 2016. Helsinki, Finland: Edita publishing Oy, 2016. Available: http://www.stat.fi/uutinen/women-and-men-infinland-2016 